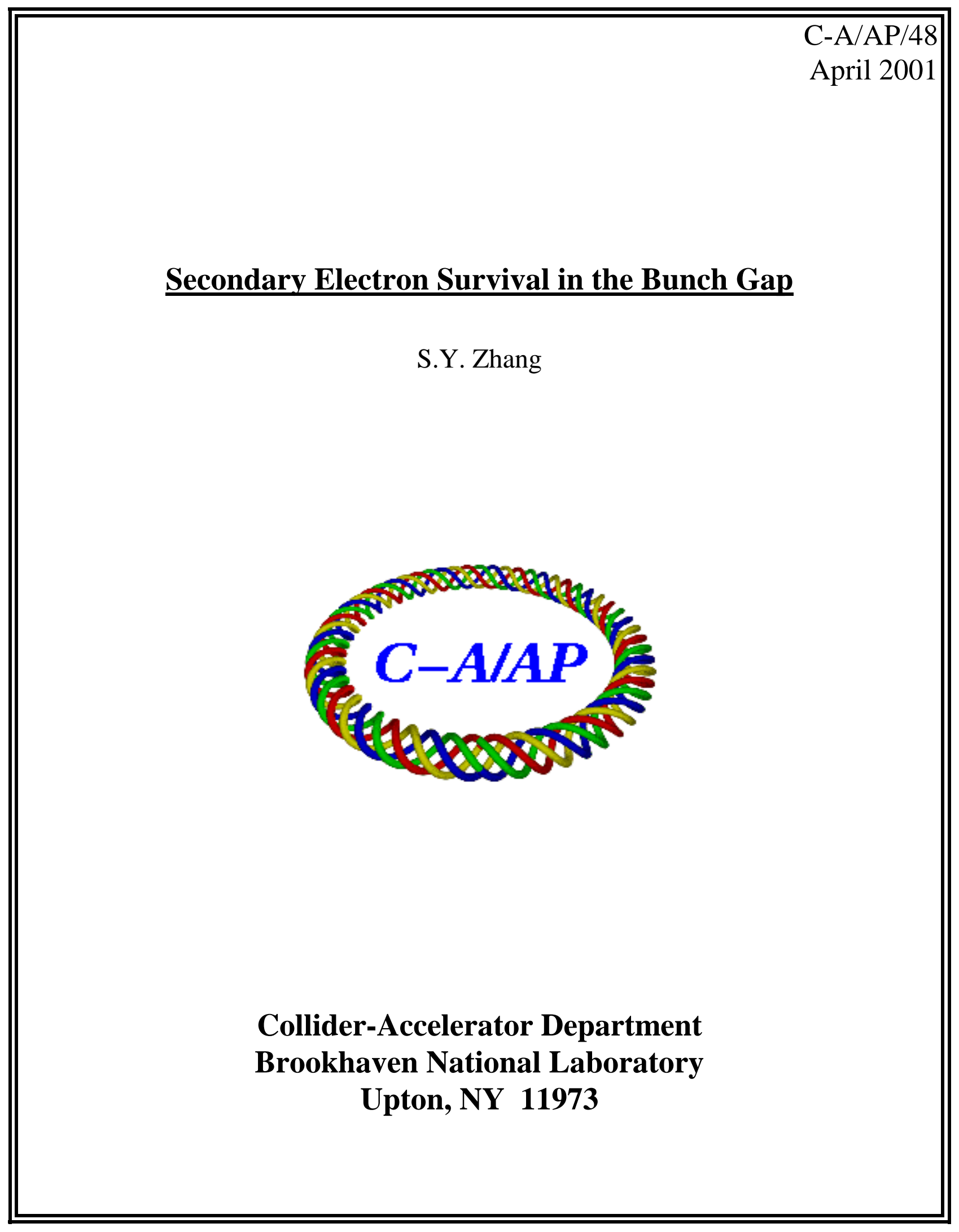




\title{
Secondary Electron Survival in the Bunch Gap
}

\author{
S.Y. Zhang
}

April 25, 2001

\begin{abstract}
One of the most important factors in the electron cloud build-up is the secondary electron (SE) survival in the bunch gap. In addition to the energy distribution, SE also has an angular distribution. In this note, the SE survival in the bunch gap is modified to include the $\mathrm{SE}$ angular distribution. It is shown that with this effect, the survival rate is substantially extended. Some related issues in concerns of the RHIC electron cloud are also discussed. For 120 bunches in the RHIC ring, the electron cloud has little chance to build-up. However, for more than 120 bunches, the chance becomes large.
\end{abstract}

\section{Introduction}

One of the most important factors in the electron-cloud build-up is the secondary electron (SE) survival in the bunch gap. For hadron colliders, such as RHIC and LHC, the typical bunch spacing is in a range of 20 ns to 200 $n s$, the SE survival rate varies in a large range, and it is usually the first issue in estimating the electron cloud threshold.

The secondary electrons have an energy distribution, most secondary electrons are at the low energy of a few $\mathrm{eV}$. Also, the emitted secondary electrons have an angular distribution, which plays an important role in the SE survival in the bunch gap.

In this note, the SE survival in the bunch gap is modified to include the $\mathrm{SE}$ angular distribution. It is shown that with this effect, the survival rate is substantially extended. Some related issues in concerns of the RHIC electron cloud are also discussed. 


\section{SE Survival Rate in the Bunch Gap}

The secondary electron energy distribution is independent of the primary electrons $(\mathrm{PE})$ or ions. In general, the following distribution function can be used [1],

$$
\frac{d N_{e}}{d E}=\sqrt{\frac{E}{E_{s m}}} \frac{N_{e}}{\sqrt{2 \pi} E_{s m}} \exp \left(-\frac{1}{2} \frac{E}{E_{s m}}\right)
$$

where $N_{e}$ is the number of electrons, and $E_{s m}$ is the peak energy of SE. The range of $E_{s m}$ for the metal surface is from 1 to $5 \mathrm{eV}$ [2].

The determination of the peak energy, which is influenced by the target material, may introduce a large difference in the estimated SE survival rate. For instance, the velocity of an electron of $5 \mathrm{eV}$ is larger than the one of 1 $\mathrm{eV}$ by a factor of larger than 2. For the surface of $\mathrm{Fe}$ and $\mathrm{Cu}$, the peak energy is $E_{s m}=2.39 \mathrm{eV}$ and $E_{s m}=3.79 \mathrm{eV}$ [2], respectively. These are consistent with the survey of numerous experiment results shown in [3]. For the stainless steel chamber, therefore, it is acceptable to use $E_{s m}=2.5 \mathrm{eV}$.

From the SE energy distribution and using $E=m_{e} v^{2} / 2$, the velocity distribution can be calculated as

$$
\frac{d N_{e}}{d v}=\left(\frac{v}{v_{s m}}\right)^{2} \frac{2 N_{e}}{\sqrt{2 \pi} v_{s m}} \exp \left(-\frac{1}{2}\left(\frac{v}{v_{s m}}\right)^{2}\right)
$$

where $m_{e}=0.511 \mathrm{MeV} / \mathrm{c}^{2}$ is the mass of electron, and $v_{s m}$ is from $E_{s m}=$ $m_{e} v_{s m}^{2} / 2$.

In Fig.1, the SE energy and velocity distributions are shown, where $E_{s m}=$ $2.5 \mathrm{eV}$ is used.

Furthermore, from the SE velocity distribution, the time needed by the electrons to cross the chamber can be calculated [1]. The distribution of this transit time is

$$
\frac{d N_{e}}{d t}=-\left(\frac{t_{s m}}{t}\right)^{4} \frac{N_{e}}{\sqrt{2 \pi} t_{s m}} \exp \left(-\frac{1}{2}\left(\frac{t_{s m}}{t}\right)^{2}\right)
$$

where $2 b=v_{s m} t_{s m}$ is the diameter of the chamber.

In addition to the energy distribution, the SE have a cosine angular distribution for the metal surface $[2,4,5]$, which is independent of the projectile incident angle. 

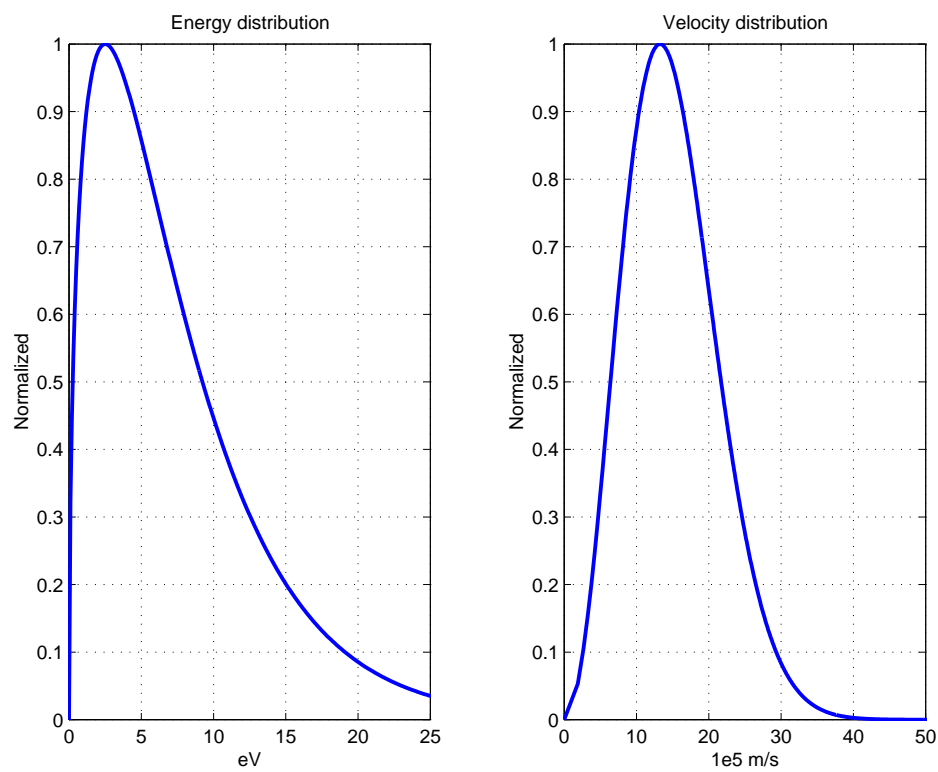

Figure 1: Secondary electron energy and velocity distributions.

Neglecting the effect of the moving frame relative to the bunch motion, the SE angular distribution can be implemented to modify the electron transit time distribution. In specific, using the transit time distribution without angular distribution, at each transit time, say $25 \mathrm{~ns}$, the distribution is extended into the region of $i 25 \mathrm{~ns}$ in a cosine fashion. The total area of this distribution at each original transit time is normalized by the original amplitude, therefore, the total number of SE of given energy keeps unchanged. In Fig.2a, the transit time distribution without angular distribution is shown. In Fig.2b, the distribution modification is illustrated for transit times spaced of 15 ns between each other.

Adding these modified functions results in the transit time distribution with the angular distribution, as that shown in Fig.2a for comparison.

Let the modified transit time distribution be $\left(d N_{e} / d t\right)_{m d}$, the secondary electron survival rate vs. the beam gap is

$$
\alpha(t)=1-\int_{0}^{t}\left(\frac{d N_{e}}{d t}\right)_{m d} d t
$$

The RHIC SE survival rate vs. the bunch gap is shown in Fig.3, where the chamber radius is $b=3.46 \mathrm{~cm}$. Note that the average chamber radius of 

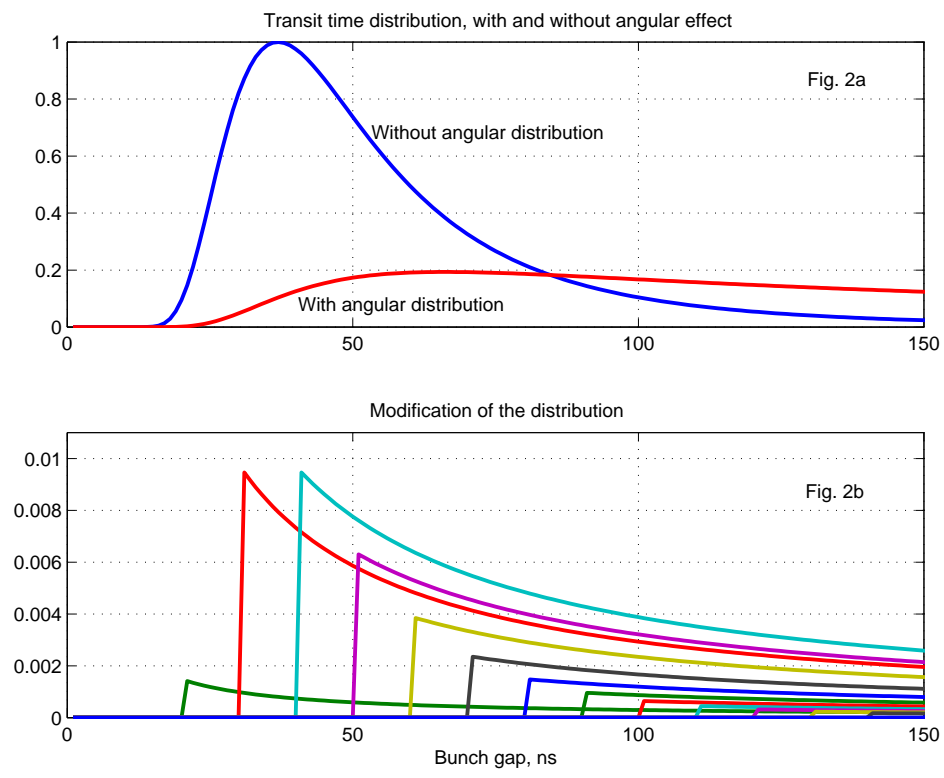

Figure 2: Secondary electron transit time distribution, and the illustration of the modification with angular distribution.

the entire ring is slightly larger than $3.46 \mathrm{~cm}$. The SE survival rate without angular distribution is also shown for comparison. It can be observed that the SE angular distribution has a large impact on the electron survival rate in the bunch gap.

\section{Discussion}

The SE survival rate in the bunch gap is larger than the conventional thinking, if the SE angular distribution is taken into account. For instance, in the case of 120 bunches in the RHIC, the bunch spacing is $106 \mathrm{~ns}$, the SE survival rate is about $75 \%$ from Fig.3. The real bunch spacing, taking off the bunch length, is smaller than $106 \mathrm{~ns}$, and hence 80\% SE could survive.

Now, let us consider the SE multipacting condition, which is $\alpha Y>1$, with $Y$ the average SE yield. According to the Seiler model, this yield is $[4,6]$, 


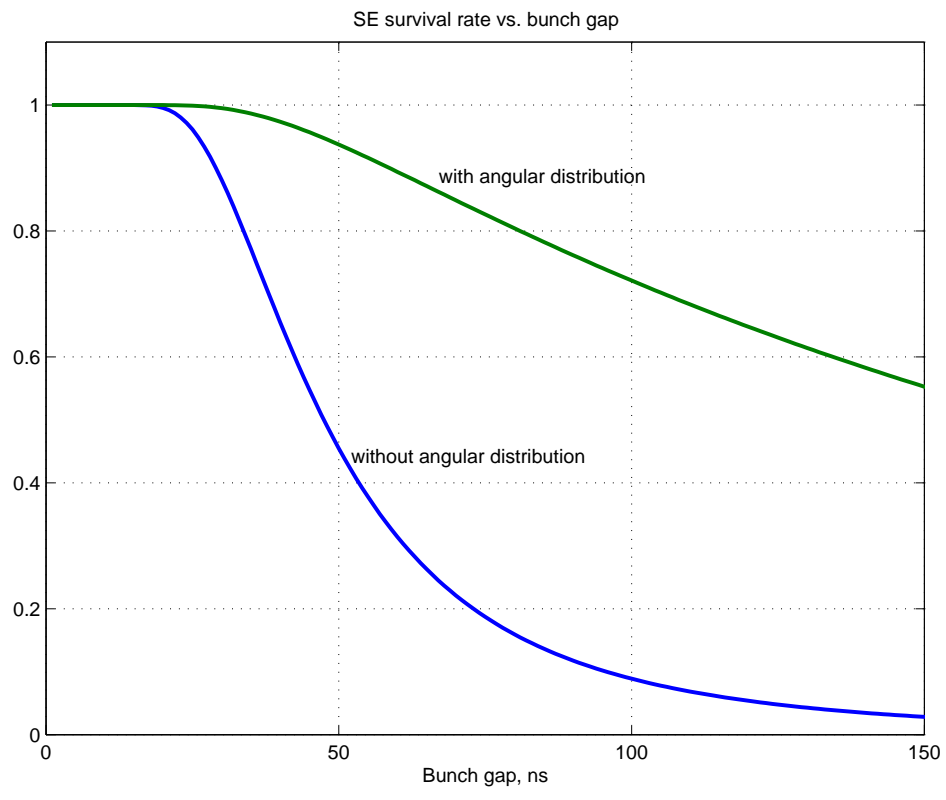

Figure 3: Secondary electron survival rate vs. bunch spacing, with and without angular distribution.

$$
Y=\delta_{\max } 1.11\left(\frac{E}{E_{p m}}\right)^{-0.35}\left(1-\exp \left(-2.3\left(\frac{E}{E_{p m}}\right)^{1.35}\right)\right)
$$

where $\delta_{\max }$ is the maximum yield, and $E_{p m}$ is the projectile (electron) energy that gives rise to maximum yield.

For stainless steel, without the scraping effect, the usual ranges are $1.2 \leq$ $\delta_{\max } \leq 1.6$, and $300 \mathrm{eV} \leq E_{p m} \leq 500 \mathrm{eV}$. Therefore, we may take $\delta_{\max }$ $=1.4$, and $E_{p m}=400 \mathrm{eV}$. The SE yield is shown in Fig.4.

The secondary electrons that gained energy in the bunch passing will plunge into the wall almost perpendicularly, therefore, the projectile incident angle effect can be neglected. Also, depending on the radial position, the energy of SE gained is ranging from zero up to the beam potential, which depends on both the beam transverse size and bunching factor, in addition to the bunch intensity. Among the SE, only those with energy from $150 \mathrm{eV}$ to $1400 \mathrm{eV}$ have SE yield larger than unity. For the SE yield shown in Fig.4, the mean yield of the SE with energy from $150 \mathrm{eV}$ to $1400 \mathrm{eV}$ is 1.2 . The mean yield of entire survived SE should be much smaller than 1.2. 


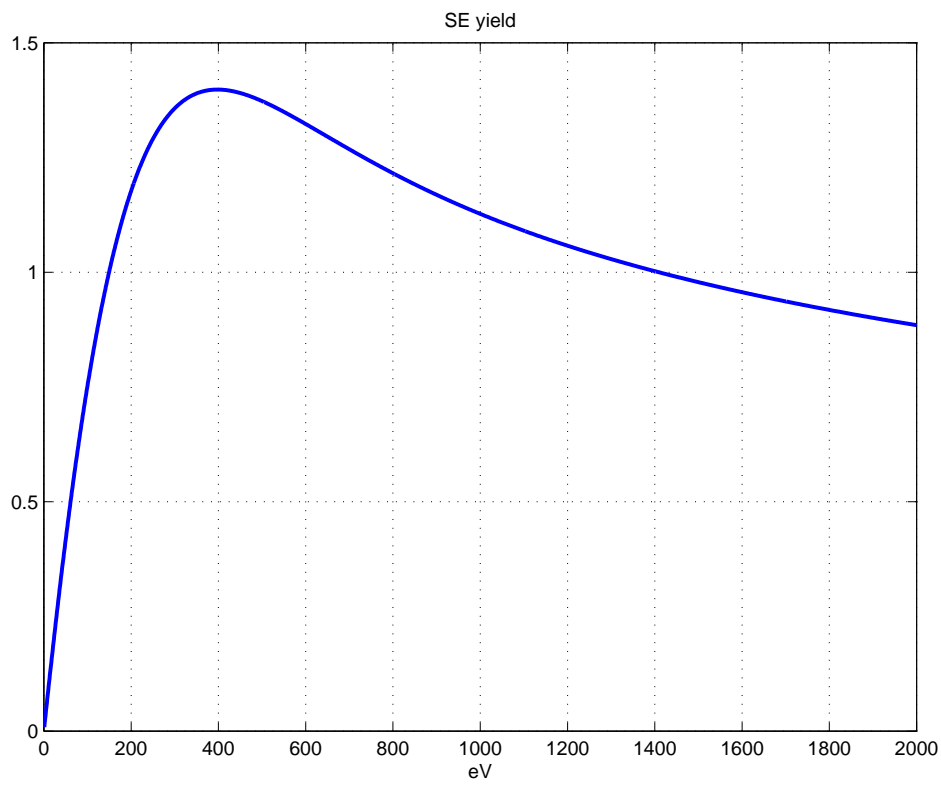

Figure 4: Secondary electron yield for stainless steel chamber.

Consider the RHIC 120 bunch with $80 \%$ SE survival during the bunch gap, the electron multipacting requires the average yield to be $\geq 1.25$, which is close to the maximum yield. Therefore, the electron cloud build-up is not likely to happen.

On the other hand, for 360 bunches, the bunch spacing is $35 \mathrm{~ns}$, and the SE survival rate is about $99 \%$. A slightly larger than unity SE average yield can create electron multipacting. Something has to be done, therefore, to suppress the electron cloud build-up.

\section{Reference}

1. L. Vos, LHC Project Note, 150, CERN, July, 1998.

2. K. Kanaya and H. Kawakatsu, J. Phys. D: Appl. Phys., Vol. 5, pp. 1727-1742, 1972.

3. J. Schou, Phys. Rev. B22, 2141, 1980.

4. H. Seiler, J. Appl. Phys. 54, R1, 1983. 
5. H. Rothard, et. al., Nucl. Instrum. Methods, B48, pp. 616-620, 1990.

6. E. Somersalo, et. al., Particle Accelerators, Vol.59, pp.107-141, 1998. 